\title{
Microbiology, Clinical Spectrum and Outcome of Peritonitis in Patients Undergoing Peritoneal Dialysis in India: Results from a Multicentric, Observational Study
}

Georgi Abraham*, Amit Gupta, Kashi Natha Prasad, Anusha Rohit, Vishwanath Billa, Rajasekhar Chakravarti, Tonmoy Das, Thadakanathan Dhinakaran, Arup Ratan Dutta, Padmanabhan Giri, Gokul nath, Tarun Jeloka, Vivekanand Jha, Sampath Kumar, Arghya Majumdar, Ajay Marwaha, Sunil Prakash S, Radha Vijay Raghavan and Rajaram KG

Madras Medical Mission, Mogappair, Chennai, India

"Corresponding author: Georgi Abraham, Madras Medical Mission, 4-A, Dr. J J Nagar, Mogappair, Chennai- 60037, India, Tel: 9841710992; E-mail: abraham_georgi@yahoo.com

Received date: April 02, 2016; Accepted date: May 10, 2016; Published date: May 17, 2016

Copyright: ( 2016 Abraham G, et al. This is an open-access article distributed under the terms of the Creative Commons Attribution License, which permits unrestricted use, distribution, and reproduction in any medium, provided the original author and source are credited.

\begin{abstract}
Background: Peritoneal dialysis related peritonitis is a major risk factor for drop out of patients on Continuous ambulatory peritoneal dialysis (CAPD) and Automated peritoneal dialysis (APD). Factors affecting PD related peritonitis and center specific microbiological data are lacking in India. A multicentric prospective observational study was designed to overcome the gaps in the existing data regarding causative organism and outcome.
\end{abstract}

Methods: The present study was a prospective, uncontrolled, open-label; observational study conducted in 21 centers representing all the four geographical regions (North, South, East and West) of India between April 2010 and December 2011.

Results: A total of 244 patients on chronic PD with peritonitis were enrolled in the study from 21 centres covering the different geographical areas of India. A total of 244 episodes (patients 244) were identified to meet the criteria for the definition of peritonitis. Climatically, $44(18.1 \%)$ episodes occurred during winter and $35(14.3 \%)$ in summer. Amongst the 85 samples that were culture positive, $38(44.7 \%)$ were in the monsoon season followed by $23(27.1 \%)$ in the postmonsoon, $18(21.2 \%)$ during winter and $11(12.9 \%)$ during summer. Maximum culture positivity $(72.7 \%)$ was observed with an automated technique. Micro-organisms could be isolated in only 85 cases (35.3\%) while the remaining samples were culture negative $(156 / 241,64.7 \%$ of samples). Organisms isolated were Gram negative in $47.8 \%$, Gram positive in $36.7 \%$, fungal in $13.3 \%$ and Mycobacterium tuberculosis in $2.2 \%$.

Conclusion: This large multicenter study of peritonitis offers insights into the etiology and outcomes of infectious complications of PD in India that are germane to clinical decision making.

Keywords: Peritonitis; Causative organisms; Seasonal variation

\section{Introduction}

In India, it is estimated that approximately 150 per million population develop end-stage renal disease (ESRD) each year which puts an enormous burden on the health care system of the country [1]. Further studies showed that approximately $90 \%$ of patients with ESRD in South Asia die within months of diagnosis because of inadequate treatment [2].

Chronic peritoneal dialysis (PD) is practiced widely in India with a penetration rate of 20 to $29 \%$ [2]. PD can be the choice of therapy in different geographical regions of the country where there is even lack of power supply and access to nephrologists is also limited [3]. PD has been advancing in terms of technique, new exchange systems, and a new generation of solutions [4]. Despite these advantages, infective complications including peritonitis (about 0.3-0.5 episodes per patient per year) remain a major cause of drop-out from PD programs. PD peritonitis is also a common cause of catheter loss and transfer to hemodialysis (HD) [5]
Multiple factors such as sociodemographic, climate change, diabetes mellitus, PD modality, and presence of peritoneal catheter tunnel infection or exit site infection are risk factors associated with the development of peritonitis. However, early diagnosis, and prompt initiation of therapy for peritonitis is critical, and can prevent adverse outcomes [6]. For an appropriate management, it is also important to identify the micro-organisms that are responsible for peritonitis $[7,8]$. Hence, the recent focus has been shifting from lowering peritonitis rates to improvement of peritonitis outcome [7-9].

Existing Indian evidence is limited to mostly being single-centered studies. There is paucity of data to guide appropriate and evidence based therapeutic choices. Hence, considering the need of a large, multi-center epidemiological data on the prevention and management of PD related peritonitis in India, the present prospective study was envisaged as the largest nationwide observational study. 
Citation: Abraham G, Gupta A, Prasad KN, Rohit A, Billa V, et al. (2016) Microbiology, Clinical Spectrum and Outcome of Peritonitis in Patients Undergoing Peritoneal Dialysis in India: Results from a Multicentric, Observational Study. J Trop Dis 4: 213. doi:10.4172/2329-891X. 1000213

Page 2 of 8

\section{Methodology}

\section{Study design and objectives}

Present study was an open-label, prospective, observational study conducted in 21 centers representing all the four geographical regions (North, South, East and West) of India, from April 2010 through December 2011. The study was approved by independent ethics committee (IEC) and written informed consent was obtained from all the study participants. The primary objective was to describe the epidemiology of etiological organisms, including the incidence and distribution of culture negative peritonitis. Secondary objectives included the assessment of clinical course and demographic risk factors, evaluation of techniques for isolation and identification of microorganism, antibiotic sensitivity of isolates, treatment protocols and peritonitis outcomes.

\section{Sample size determination}

Sample size calculation was based on a previous Indian study, in which Gram negative, Gram positive, polymicrobial and fungal peritonitis were found to be 42.45, 28.30, 10.38 and $18.87 \%$ respectively [10]. Weighted percentage peritonitis of $30.67 \%$ was calculated. Based on the anticipated peritonitis of $30.67 \%$, 95\% level of confidence and $7 \%$ margin of absolute error, a sample of 168 episodes of peritonitis was calculated. To compensate for variations between and within centers, the calculated sample was multiplied by a factor of two (design effect), giving the revised sample size of 336. Considering the dropout rate of $20 \%$, sample size was finally adopted as 400 episodes of peritonitis.

\section{Study population}

Inclusion criteria: The present study included patients who have been diagnosed to have peritonitis following Peritoneal Dialysis. PD peritonitis was defined as the presence of two of the three ISPD 2010 peritonitis diagnostic criteria [8].

Exclusion criteria: Patients with a history of peritonitis in past one month or those who received antibiotics within 7 days prior to enrolment (without an exit site infection) or concurrent presence of any malignancy or on immunosuppressants (except topical steroid) were excluded from the study.

\section{Study procedure}

Eligible participants were recruited and the following information was collected at baseline.

\section{Demographic}

2. Clinical characteristics

3. Type of peritonitis

4. Time taken for developing the current peritonitis

5. Catheter implantation technique,

6. Pre and post catheter exit site care protocol,

7. Break-in period

8. Type of dialysis solution (glucose and non-glucose based)

9. Visual analogue scale (vas) from 0-10 (in case of abdominal pain)]
10. Laboratory data (cell count and differential count of dialysate effluent, gram stain, culture and sensitivity of dialysate effluent, complete blood count, serum albumin) was recorded at baseline.

11. Variation as per seasons (Winter-December to February; Summer-March to May; Monsoon-June to September; Post-monsoonOctober and November) of the episodes was also assessed.

\section{Follow-up visits, Data Collection, Treatment}

Empirical antibiotics were started as per center specific protocols and were thereafter revised based on culture sensitivity reports. All the study participants were followed up on day $3 \pm 1$ (visit 2), $5 \pm 1$ (visit 3), $7 \pm 1$ (visit 4), $14 \pm 2$ (visit 5) and 45+7 (visit 6). All the above mentioned information except for microbiological investigations were collected at all these time points. Based on investigator discretion, a repeat culture was also done at visit 5 . Peritonitis related deaths in the present study was defined as death due to sepsis, death occurring with a positive peritoneal dialysis culture, death within 14 days after onset of peritonitis or death occurring during hospitalization for any patient admitted with peritonitis [10].

\section{Culture techniques}

Being an observational study, no particular culture method was made mandatory, although all the study centers were advised to use the methods recommended in ISPD 2010 guidelines [8]. Both conventional (agar and broth method) and automated culture methods (e.g., BACTEC, Septi-Chek, BacT/Alert) were permitted due to limitations of cost and resources at different centers. Automated methods are blood culture systems which process test samples and analyze results to data, and generate an interpretation using computing programs. Conventional method involved manual inoculation and enrichment step followed by plating with selective media. Prior to and during the study, meetings and training programmes were conducted to spread the awareness of proper culture techniques across the participating centers to optimize the chances of successful isolation and identification of etiological microorganisms.

\section{Statistical analysis}

The frequency distribution of some quantitative variables such as age, height, BMI, socio-economic status was classified into different class-intervals. The qualitative variables such as education, employment, marital status, gender, hygiene, geographical location, source of water, co-morbidity were expressed in terms of percentage. Percentage of peritonitis caused by Gram positive, Gram negative organisms, Mycobacterium tuberculosis, fungus and of culture negative peritonitis were estimated. All the quantitative variables were assessed for normality and accordingly student's t-test was employed. The categorical variables were represented in proportions and analysed using Chi-square test after checking for assumptions. A value of $\mathrm{p}<0.05$ was treated as significant for all the statistical tests.

\section{Results}

\section{Demographic data}

A total of 244 patients with peritonitis were enrolled in the study. The mean (SD) age (years) was 54.30 (13.1) \& body mass index $(\mathrm{kg} / \mathrm{m} 2)$ of the study participants was 23.15 (4.5) respectively. The median (range) duration (years) of ESRD was 2.75 (0.05-18.87) and that of peritoneal catheterization was $1.26(0.03-15.62)$ respectively. A 
Citation: Abraham G, Gupta A, Prasad KN, Rohit A, Billa V, et al. (2016) Microbiology, Clinical Spectrum and Outcome of Peritonitis in Patients Undergoing Peritoneal Dialysis in India: Results from a Multicentric, Observational Study. J Trop Dis 4: 213. doi:10.4172/2329-891X. 1000213

Page 3 of 8

total of 61 (25\%) participants were new to PD and 177 (72.5\%) had undergone a previous hemodialysis while $6(2.5 \%)$ had a failed previous kidney transplant. Of the 244 participants, 239 (98\%) were on continuous ambulatory peritoneal dialysis (CAPD) mode with a median (range) duration (months) of $15.76(0.4-187.3)$ and only 5 $(2.0 \%)$ were on automated peritoneal dialysis (APD) with a median duration (months) of 7.13 (4.7-12.3). Table 1 describe the demographic characteristics of the study participants

\begin{tabular}{|c|c|c|c|}
\hline Variables & Attributes & $\mathrm{N}$ (Frequency) & $\%$ \\
\hline \multirow[t]{2}{*}{ Gender distribution } & Male & 160 & 65.6 \\
\hline & Female & 84 & 34.4 \\
\hline \multirow[t]{3}{*}{ Age } & $<40$ years & 28 & 11.5 \\
\hline & 40- 60 years & 148 & 60.7 \\
\hline & $>60$ Years & 68 & 27.9 \\
\hline \multirow[t]{4}{*}{ Educational status } & No formal education & 27 & 11.1 \\
\hline & Primary school & 56 & 23.0 \\
\hline & High school & 70 & 28.7 \\
\hline & College and above & 91 & 37.3 \\
\hline \multirow[t]{2}{*}{ Distance of residence from clinic } & Same city & 135 & 55.3 \\
\hline & Outside city & 109 & 44.7 \\
\hline \multirow[t]{2}{*}{ Location } & Rural & 72 & 29.5 \\
\hline & Urban & 172 & 70.5 \\
\hline \multirow[t]{5}{*}{ Personal hygiene } & Daily bath & 225 & 92.2 \\
\hline & Hand wash before and after food & 231 & 94.7 \\
\hline & Hand wash after defecation & 237 & 97.1 \\
\hline & Hand wash after urination & 186 & 76.2 \\
\hline & Clean clothes & 229 & 93.9 \\
\hline \multirow[t]{2}{*}{ Toilet habits } & Open field defecation & 1 & 0.4 \\
\hline & Sanitary toilets & 243 & 99.6 \\
\hline \multirow[t]{4}{*}{ Water source } & Tap water & 213 & 87.3 \\
\hline & Tube well & 56 & 23.0 \\
\hline & Well water & 10 & 4.1 \\
\hline & River & 2 & 0.8 \\
\hline \multirow[t]{5}{*}{ Socioeconomic status (Income in Rs/month) } & $<5,000$ & 22 & 9.0 \\
\hline & $5,000-10,000$ & 41 & 16.8 \\
\hline & $10,000-20,000$ & 60 & 24.6 \\
\hline & $>20,000$ & 48 & 19.7 \\
\hline & Do not wish to disclose & 73 & 29.9 \\
\hline
\end{tabular}

Table 1: Attributes of the patients undergoing peritoneal dialysis $(\mathrm{N}=244)$.

Reviewing the clinical consideration and presence of comorbid disease, total of 106 (43.4\%) patients had diabetic nephropathy, 96 (39.3\%) had hypertensive nephropathy. The remaining 42 (17.3\%) had underlying chronic glomerulonephritis, chronic interstitial nephritis, obstructive nephropathy, toxic nephropathy, polycystic kidney disease, renovascular disease. 
Citation: Abraham G, Gupta A, Prasad KN, Rohit A, Billa V, et al. (2016) Microbiology, Clinical Spectrum and Outcome of Peritonitis in Patients Undergoing Peritoneal Dialysis in India: Results from a Multicentric, Observational Study. J Trop Dis 4: 213. doi:10.4172/2329-891X. 1000213

Page 4 of 8

\section{PD Catheterization}

Pre catheter insertion and exit site preparation was performed in 237 (97.1\%) patients. Tenckhoff swan neck double cuff catheters and straight catheters were used in $158(64.8 \%)$ and $86(35.2 \%)$ patients respectively. The twin bag disconnected system was used in $239(98 \%)$ patients. Post catheterization, topical antibiotics (mupirocin $98.1 \%$ \& povidone iodine $1.9 \%$ ) was used in $213(87.3 \%)$ patients at exit site. The median duration (days) of peritoneal rest before utilization was 12 (0-30) days, and only 86/244 (35.2\%) patients had a break in period following dialysis of 14 days as per ISPD guidelines.

Glucose based dialysis fluid was used in most of the cases (90.2\%) as compared to non-glucose based dialysis fluid. Both were used in 22/244 (9.0\%) patients. Although the number of PD exchanges ranged from 1 to 5 times daily, majority of the patients 189/244 (77.5\%) had undergone $3 \times 2 \mathrm{~L}$ exchanges per day. 185 patients, $(75.8 \%)$ had $6 \mathrm{~L}$ of fluid exchanges per day which was performed by caretakers in $81.1 \%$ of the cases. A recent change in the caretaker was reported in 23/244 (11.6\%) of which only 17/23 (73.9\%) had a formal training in PD care.

\section{Previous episodes, latency and seasonality of peritonitis}

There were 159 (65.2\%) patients who never had any episode of peritonitis in the past, while $53(21.7 \%)$ recorded the second episode, $21(8.6 \%)$ recorded the third episode, 7 (2.9\%) patients recorded the fourth episode and $4(1.6 \%)$ patients demonstrated the fifth episode. The median time (months) from first catheterization to first episode of peritonitis was $8.9(0.4-112.1)$, and corresponding time to their most recent episode of peritonitis was 12 (1-108) months. Table 2 compares the time taken from catheterization to first episode of peritonitis, last episode of peritonitis and the time gap between last episode of peritonitis and the current episode. We have observed $110(45.1 \%)$ patients develop peritonitis episodes in the monsoon season followed by $55(22.5 \%)$ in post-monsoon period. A total of 44/244 (18.1\%) episodes occurred during winter and 35/244 (14.3\%) in summer. Only 1 patient reported exit site infection while none reported tunnel infection. Table 3 depicts the monthly distribution of peritonitis, micro-organism wise and their outcomes.

\begin{tabular}{|c|c|c|c|}
\hline & First peritonitis time ${ }^{*}$ & Last peritonitis time** & Last peritonitis time $2^{\star \star \star}$ \\
\hline$N$ & 244 & 85 & 85 \\
\hline Mean & 13.4 & 18.8 & 18.5 \\
\hline Median & 8.9 & 12.0 & 11.9 \\
\hline $\begin{array}{l}{ }^{*} \text { duration } \\
{ }^{* *} \text { duratio } \\
\text { ***durati }\end{array}$ & $\begin{array}{l}n \text { to first episode of perito } \\
\text { on to last episode of peritt } \\
\text { tonitis to current episode }\end{array}$ & & \\
\hline
\end{tabular}

Table 2: Time to appearance of peritonitis (months).

\begin{tabular}{|c|c|c|c|c|c|c|c|c|c|c|c|c|c|}
\hline \multicolumn{2}{|c|}{ Strain and microorganism isolated } & \multicolumn{12}{|c|}{ No. of Peritonitis Episodes due to microorganism (1 and 2) due to seasonal variation } \\
\hline & & Jan & Feb & Mar & Apr & May & Jun & Jul & Aug & Sep & Oct & Nov & Dec \\
\hline \multirow[t]{5}{*}{ Gram positive } & $\begin{array}{ll}\text { Coagulase } & \text { Negative } \\
\text { Staphylococci (16) } & \end{array}$ & 0 & 1 & 1 & 0 & 2 & 1 & 2 & 2 & 3 & 0 & 3 & 1 \\
\hline & Staphylococcus Aureus (10) & 2 & 0 & 0 & 1 & 0 & 1 & 0 & 0 & 3 & 1 & 1 & 1 \\
\hline & Streptococcus sps (5) & 1 & 0 & 0 & 1 & 1 & 0 & 1 & 0 & 0 & 1 & 0 & 0 \\
\hline & Enterococcus Sps (2) & 0 & 0 & 0 & 0 & 0 & 1 & 0 & 0 & 0 & 0 & 1 & 0 \\
\hline & $\begin{array}{l}\text { Total episodes by gram positive } \\
\text { species (33) }\end{array}$ & 3 & 1 & 1 & 2 & 3 & 3 & 3 & 2 & 6 & 2 & 5 & 2 \\
\hline \multirow[t]{8}{*}{ Gram negative } & AcinetobacterSps (4) & 1 & 1 & 0 & 0 & 0 & 0 & 0 & 1 & 0 & 0 & 1 & 0 \\
\hline & E.Coli (18) & 1 & 0 & 0 & 1 & 0 & 3 & 4 & 0 & 2 & 3 & 2 & 2 \\
\hline & EnterobacterSps(4) & 0 & 0 & 0 & 0 & 0 & 0 & 1 & 1 & 0 & 0 & 2 & 0 \\
\hline & Klebsiella pneumonia (9) & 1 & 0 & 0 & 0 & 0 & 0 & 1 & 3 & 1 & 0 & 1 & 2 \\
\hline & Alcaligenes (1) & 0 & 0 & 0 & 0 & 1 & 0 & 0 & 0 & 0 & 0 & 0 & 0 \\
\hline & Pseudomonas sps(6) & 0 & 0 & 1 & 0 & 1 & 1 & 0 & 0 & 1 & 0 & 2 & 0 \\
\hline & Oligellaureolytica(1) & 0 & 0 & 0 & 0 & 0 & 0 & 0 & 0 & 0 & 0 & 1 & 0 \\
\hline & $\begin{array}{l}\text { Total episodes by gram negative } \\
\text { species ( } 43 \text { ) }\end{array}$ & 3 & 1 & 1 & 1 & 2 & 4 & 6 & 5 & 4 & 3 & 9 & 4 \\
\hline
\end{tabular}


Citation: Abraham G, Gupta A, Prasad KN, Rohit A, Billa V, et al. (2016) Microbiology, Clinical Spectrum and Outcome of Peritonitis in Patients Undergoing Peritoneal Dialysis in India: Results from a Multicentric, Observational Study. J Trop Dis 4: 213. doi:10.4172/2329-891X. 1000213

Page 5 of 8

\begin{tabular}{|l|l|l|l|l|l|l|l|l|l|l|l|l|l|}
\hline Fungal & Candida Sps (12) & 1 & 0 & 0 & 1 & 0 & 0 & 1 & 0 & 2 & 2 & 2 & 3 \\
\hline Mycobacterium tuberculosis (2) & 0 & 0 & 0 & 0 & 0 & 0 & 0 & 0 & 2 & 0 & 0 & 0 \\
\hline Episodes reported in each month & 7 & 2 & 2 & 4 & 5 & 7 & 10 & 7 & 14 & 7 & 16 & 9 \\
\hline \multirow{3}{*}{ Outcomes } & Recovery from peritonitis & 7 & 5 & 7 & 9 & 11 & 14 & 19 & 22 & 20 & 26 & 17 & 14 \\
\cline { 2 - 12 } & Infection on-going & 1 & 0 & 1 & 0 & 0 & 1 & 2 & 1 & 0 & 2 & 0 & 0 \\
\cline { 2 - 12 } & Switch to modality & 3 & 2 & 1 & 2 & 1 & 5 & 4 & 4 & 6 & 3 & 6 & 10 \\
\cline { 2 - 12 } & Lost to follow up & 0 & 1 & 0 & 0 & 0 & 0 & 2 & 1 & 0 & 0 & 0 & 0 \\
\cline { 2 - 12 } & Death & 0 & 0 & 0 & 1 & 2 & 0 & 5 & 1 & 3 & 0 & 1 & 1 \\
\hline
\end{tabular}

Table 3: Peritonitis episodes and outcome parameters with seasonal variation.

\section{Sample transfer and techniques}

164 samples $(67.2 \%)$ were brought to the laboratory within two hours of sample collection. Of the 85 culture positive samples, 62 $(72.3 \%)$ reached the laboratory within two hours of collection. However, $52(21.3 \%)$ samples were stored at $4^{\circ} \mathrm{C}$, of which only 18 (34.6\%) were culture positive. Conventional method was used in 183 (75.0\%) patients while automated method was used in 58 (23.8\%) patients. Regarding the various techniques that were used in various centers, maximum culture positivity $(72.7 \%, 8 / 11)$ was observed with an automated technique, where sediment after centrifugation at 3000 rpm (15 minutes) of $50 \mathrm{ml}$ sample from whole bag was inoculated in an automated culture bottle and processed.

\section{PD Effluent profile \& Microbiology}

At baseline, only one patient had a visibly clear effluent PD bag, while remaining individuals had cloudy effluent. At presentation, there was increased white blood cell (WBC) count in dialysate with a median (range) count of $1200(45-31600)$ cells $/ \mathrm{mm}^{3}$. Data was unavailable for $13(5.3 \%)$ samples. The data on differential leukocyte count (DLC) showed a predominance of neutrophils [Median (range) (\%) - 88 (0-100)]. Culture \& sensitivity was performed using conventional and automated methods in $183(75.0 \%)$ and $58(23.8 \%)$ patients respectively.

A total of 90 microorganisms were isolated from 85 (35.3\%) samples in our study. The remaining $156(64.7 \%)$ samples were culture negative. Within the culture positive samples, $80(94.1 \%)$ samples demonstrated single micro-organism and 5 (5.9\%) showed two micro-organisms. Automated culture technique was used in $44.8 \%$ culture positive cases as compared to $32.2 \%$, using conventional method $(\mathrm{P}=0.08)$. We found $43(47.8 \%)$ Gram negative and 33 (36.7\%) Gram positive microorganism in our sample respectively. However, remaining 12 (13.3\%) episodes were caused by fungi and $2(2.2 \%)$ were due to Mycobacterium tuberculosis. The predominant micro-organism that was grown included Escherichia coli (20\%) followed by Coagulase negative Staphylococcus spp (17.8\%) and Candida species (13.3\%).

\section{Antibiotics for management of peritonitis}

As a prophylactic care during catheter implantation, antibiotics were administered to 243 (99.6\%) patients. The most commonly used antibiotic was vancomycin $1 \mathrm{gm}(35.4 \%)$. Cefazolin (30.9\%) and tobramycin (16.5\%) was the other most commonly used antibiotic.

\section{Clinical outcome}

Of the total 244 patients with peritonitis, 6 dropped out after first visit. A total of 179 participants completed the study. Figure 1 depicts the patient flow from the time of recruitment till the completion of the last study visit. In the last study visit period, there were a total of 13 participants with peritonitis. PD effluent cell count was analysed only for these participants and was found to be elevated. Among 179 (73.4\%) patients, $171(95.5 \%)$ patients recovered from peritonitis while 8/179 (4.5\%) did not recover. Overall, 14 (5.7\%) patients died during the study period, 51/244 (20.9\%) patients dropped out from the study, $47 / 244$ (19.3\%) patients switched to another modality (hemodialysis or renal transplant), and 4/244 (1.6\%) patients were lost to follow up. The reasons for the loss to follow up were the lower socio-economic status, literacy, distance from center etc.

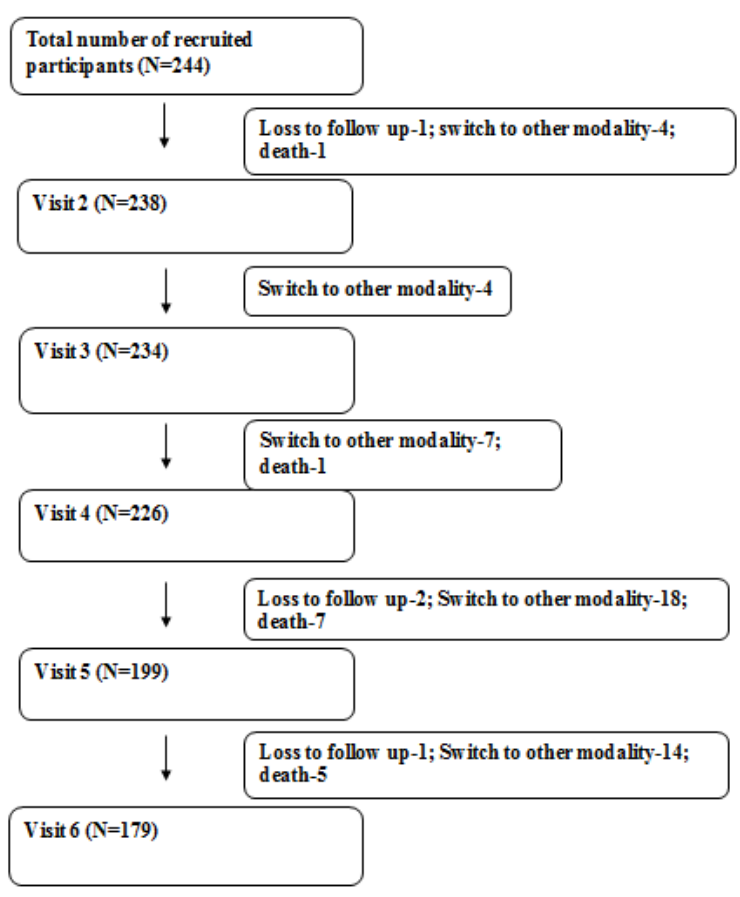

Figure 1: Flowchart of the study participants. 
Citation: Abraham G, Gupta A, Prasad KN, Rohit A, Billa V, et al. (2016) Microbiology, Clinical Spectrum and Outcome of Peritonitis in Patients Undergoing Peritoneal Dialysis in India: Results from a Multicentric, Observational Study. J Trop Dis 4: 213. doi:10.4172/2329-891X. 1000213

Page 6 of 8

The clinical outcomes were similar in both culture positive and negative peritonitis patients. Recovery from peritonitis was seen in $68.2 \%$ of culture positive cases and $71.1 \%$ culture negative patients. In addition, $20 \%$ and $18.8 \%$ patients were switched to either hemodialysis or renal transplantation, $9.4 \%$ and $3.8 \%$ died, $1.2 \%$ and $4.4 \%$ had ongoing infections and $1.2 \%$ and $1.9 \%$ was lost to follow up in culture positive and culture negative group.

Further we found that, out of 18 patients with E.coli infection, 12 (66.7\%) recovered from peritonitis, 1 (5.6\%) switched to hemodialysis and $5(27.8 \%)$ died. Mortality was also recorded in $2 / 8(20 \%)$ who grew Staphylococcus aureus, 1 each with Acinetobacter $s p .(\mathrm{n}=3)$ and Mycobacterium tuberculosis $(\mathrm{n}=1)$ died. The best outcomes were observed in patients with peritonitis caused by Coagulase negative staphylococci $(\mathrm{n}=16)$, in which $14(87.5 \%)$ patients recovered from peritonitis, $2(12.5 \%)$ switched to other modality, while none died as shown in Table 4. Most of the individuals infected with Candida sp. $(n=12)$ had switch to other modalities of renal replacement therapy following catheter removal $(9,75 \%)$ after a median duration of 14 days. E.coli, Coagulase negative Staphylococci, Candida Sp., Klebsiella pneumonia and Staphylococcus aureus were the most frequent microorganisms responsible for hospitalization of the patients. Statistical correlations were not made due to less number in each individual group.

\begin{tabular}{|c|c|c|c|c|c|c|c|c|}
\hline \multicolumn{2}{|c|}{ Strain and microorganism isolated } & \multirow{2}{*}{$\begin{array}{l}\begin{array}{l}\text { Number } \\
\text { microorganism }\end{array} \\
16\end{array}$} & \multirow{2}{*}{$\begin{array}{l}\%(\mathrm{~N}=90) \\
17.77\end{array}$} & \multirow{2}{*}{$\begin{array}{l}\text { Recovery from } \\
\text { Peritonitis } \\
(\mathrm{N}=171) \%\end{array}$} & \multirow{2}{*}{  } & \multirow{2}{*}{$\begin{array}{l}\text { Modality } \\
\text { Switch } \\
(\mathrm{N}=47) \\
2\end{array}$} & \multirow{2}{*}{$\begin{array}{l}\text { Lost } \\
\text { Follow-up } \\
(\mathrm{N}=4) \\
0\end{array}$} & \multirow{2}{*}{$\begin{array}{l}\text { Death } \\
(\mathrm{N}=14)\end{array}$} \\
\hline $\begin{array}{l}\text { Gram } \\
\text { positive }\end{array}$ & $\begin{array}{ll}\text { Coagulase } & \text { Negative } \\
\text { Staphylococci } & \end{array}$ & & & & & & & \\
\hline & Staphylococcus aureus & 10 & 11.11 & 8 & 0 & 1 & 0 & 2 \\
\hline & Streptococcus sps & 5 & 5.55 & 4 & 0 & 0 & 1 & 0 \\
\hline & Enterococcus Sps & 2 & 2.22 & 1 & 0 & 1 & 0 & 0 \\
\hline \multicolumn{4}{|c|}{ Total gram positive cases $(33)$} & $27(81.8 \%)$ & 0 & $4(12.1 \%)$ & $1(3 \%)$ & $2(6.1 \%)$ \\
\hline \multirow{7}{*}{$\begin{array}{l}\text { Gram } \\
\text { negative }\end{array}$} & AcinetobacterSps & 4 & 4.44 & 3 & 0 & 0 & 0 & 1 \\
\hline & E.Coli & 18 & 20.00 & 12 & 0 & 1 & 0 & 5 \\
\hline & EnterobacterSps & 4 & 4.44 & 4 & 0 & 0 & 0 & 0 \\
\hline & Klebsiella pneumonia & 9 & 10.00 & 6 & 0 & 2 & 0 & 0 \\
\hline & Alcaligenes & 1 & 1.11 & 1 & 0 & 0 & 0 & 0 \\
\hline & Pseudomonas sps & 6 & 6.66 & 5 & 1 & 0 & 0 & \\
\hline & Oligellaureolytica & 1 & 1.11 & 0 & 0 & 1 & 0 & 0 \\
\hline \multicolumn{4}{|c|}{ Total gram negative cases (43) } & $31(72.1 \%)$ & $1(2.3 \%)$ & $4(9.3 \%)$ & 0 & $6(13.9 \%)$ \\
\hline Fungal & Candida Sps & 12 & 13.33 & 3 & 0 & 9 & 0 & 0 \\
\hline \multicolumn{2}{|c|}{ Mycobacterium tuberculosis } & 2 & 2.22 & 1 & 0 & 0 & 0 & 1 \\
\hline \multicolumn{2}{|l|}{ Total } & 90 & 100 & & & & & \\
\hline
\end{tabular}

Table 4: The best outcomes were observed in patients with peritonitis caused by Coagulase negative staphylococci.

\section{Discussion}

The present study is the largest multi-centre nation-wide study from India reporting the pattern and outcomes of peritonitis from various geographical regions of the country. A proper understanding of the patterns of occurrence of peritonitis in patients on $\mathrm{PD}$, their natural history in the course of therapy, and the various predisposing factors that identify patients at risk is crucial to therapeutic and prophylactic interventions [11].

The present study demonstrated a very high culture negativity rate $(65.3 \%)$, value far greater than that suggested in ISPD guidelines $(<20 \%)$. A culture negativity rate between $28-70 \%$ has been observed in several studies with conventional culture technique [10,12-14]. Nonadherence to ISPD recommendations with regard to culture specimen collection and processing methods along with discordance in sampling technique might have resulted in high culture negativity rates. A study from South India had a culture negativity rate of 50\% even with the use of automated system [7]. Similar to this, we also found that when culture and sensitivity testing was done by automated technique, nearly $45 \%$ of the samples showed a growth of micro-organisms as against 32\% with the conventional techniques. Reason for culture negative peritonitis can be attributed to either use of conventional techniques versus automation, use of substandard media for culture especially in the public sector hospitals and sub-optimal microbiological techniques. However, the number of samples was small to draw firm conclusions.

Increased incidence of Gram negative organisms, unlike the western literature, has been encountered in other studies in India $[10,14]$. 
Citation: Abraham G, Gupta A, Prasad KN, Rohit A, Billa V, et al. (2016) Microbiology, Clinical Spectrum and Outcome of Peritonitis in Patients Undergoing Peritoneal Dialysis in India: Results from a Multicentric, Observational Study. J Trop Dis 4: 213. doi:10.4172/2329-891X. 1000213

Page 7 of 8

Sharma et al. (2003) from North India had shown a predominance of Gram-negative organisms (60-66\%) with E. coli being most frequently isolated $[12,13,15]$. Gupta et al. (2011) reported higher incidence of Gram-positive (55.5\%) than Gram negative organisms (45\%) in South India [7]. In our study, $47.8 \%$ Gram negative and $36.7 \%$ Gram positive microorganism were isolated respectively. E. coli was the most common (20\%) followed by Coagulase Negative Staphylococci (17.7\%) of the total microorganism isolated. The incidence of fungal peritonitis in the present study (13.3\%) was found to be similar to previous studies from India $[7,13,16]$. Lo et al. found that use of antifungal prophylaxis during any antibiotic therapy resulted in decreased risk of Candida peritonitis [17]. Hospitalization was necessary in about $71 \%$ of total peritonitis episodes in this study, which is consistent with the literature. The data is similar to that of a study by Ghali et al. wherein hospitalization occurred for $70 \%$ of total episodes [18].

Proper treatment is crucial for favorable outcome of PD related peritonitis. Inadequate culture facilities in many hospitals are contributing to the need for broad spectrum empirical antibiotics, which are expensive and drives the emergence of antimicrobial resistance [19]. Though initial empirical antibiotic prescription is recommended, it is generally expected that local epidemiology and sensitivity pattern should guide the appropriate antibiotic selection. According to ISPD guidelines also, the initial treatment of peritonitis (prior to the results of microbiological tests) should be based on a combination of drugs for coverage of gram-positive cocci and gramnegative organisms [6]. In the present study, vancomycin (54.9\%), cefazolin (43.4\%) and ceftazidime (42.2\%) were the most frequently prescribed antimicrobials. Gram-positive organisms may respond to vancomycin or a cephalosporin, and Gram-negative organisms to a third generation cephalosporin or aminoglycoside.

Although ISPD guidelines recommend a beta-lactum and use of vancomycin to be restricted to patients with methicillin-resistant Staphylococcus aureus, we found vancomycin being the preferred empirical antimicrobial in our study [6]. The choice of empiric outpatient treatment is dictated by convenience of self-administration by the patient and by the drug's pharmacokinetics, its cost, and its effectiveness. The pharmacokinetics of vancomycin allows IP dosing at weekly intervals [20]. Additionally, Santoianni et al. recommended vancomycin plus ceftazidime or imipenem as the empirical antibiotics in the long term study [21]. A study which compared the efficacy of vancomycin versus cefazolin in PD peritonitis patients found that patient compliance and satisfaction was better with vancomycin than cefazolin when used as empirical antimicrobial [22].

In our study, 171 (95.5\%) patients recovered from peritonitis at the end of trial. The clinical recovery was similar in both culture positive (68.2\%) and negative (71.1\%) peritonitis. Recovery from peritonitis was higher in gram positive than gram negative peritonitis and the mortality rate was higher for Gram-negative than for Gram-positive infections, which is similar to other reports including from India [14,23-25]. High recovery rate may point towards the proper and timely use of empirical antibiotics and later supported by culture and antibiotic susceptibility results. Also continuous review of every episode of infection and timely change of interventions may have helped to improve outcomes.

However, this being an observational study has its own limitations. Observational studies are not scientifically capable of proving or disproving hypothesis. The same also applies to this epidemiological study because of the heterogeneity of real-life patient populations, the lack of standardised culture methods, treatment regimens and standardised indications for change of treatment. Also the total sample could not be recruited despite extending the enrolment period.

\section{Conclusion}

This first report from a large database offers insight into the etiology and outcomes of PD related peritonitis in India, which are germane to clinical decision making. The study has described the demography, microbiology, treatment and outcomes of peritonitis in Indian PD patients. Information regarding the seasonal trend and outcomes of peritonitis based on causative organism may help to identify patients with poor prognosis and take preventive and proper therapeutic measures. Much remains to be learned, and several areas require further research. A more detailed knowledge of the reasons for high culture negativity and resistance profiles of the causative organisms is needed to guide appropriate antibiotic therapy and improve peritonitis outcome.

\section{References}

1. Limesh M, Annigeri RA, Mani MK, Kowdle PC, Rao BS, et al. (2012) Retarding the progression of chronic kidney disease with renin angiotensin system blockade. Indian J Nephrol 22: 108-115.

2. Abraham G (2008) The challenges of renal replacement therapy in Asia. Nat Clin Pract Nephrol. 4: 643.

3. Abraham G, Varughese S, Mathew M, Vijayan M (2015) A review of acute and chronic peritoneal dialysis in developing countries. Clin Kidney J 8: 310-317.

4. Tokgoz B (2009) Clinical advantages of peritoneal dialysis. Perit Dial Int 29 Suppl 2: S59-61.

5. Quintanar Lartundo JA, Palomar R, Dominguez-Diez A, Salas C, RuizCriado J, et al. (2011) Microbiological profile of peritoneal dialysis peritonitis and predictors of hospitalization. Adv Perit Dial 27: 38-42.

6. Piraino B, Bailie GR, Bernardini J, Boeschoten E, Gupta A, et al. (2005). Peritoneal dialysis-related infections recommendations: 2005 update. Perit Dial Int 25: 107-131.

7. Gupta S, Muralidharan S, Gokulnath, Srinivasa H (2011) Epidemiology of culture isolates from peritoneal dialysis peritonitis patients in southern India using an automated blood culture system to culture peritoneal dialysate. Nephrology (Carlton) 16: 63-67.

8. Li PK, Szeto CC, Piraino B, Bernardini J, Figueiredo AE, et al. (2010) ISPD guidelines/recommendations. Peritoneal Dialysis Related Infections Recommendations: 2010 update. Perit Dial Int 25: 107-131.

9. Van Esch S, Krediet RT, Struijk DG (2012) Prognostic factors for peritonitis outcome. Contrib Nephrol 178: 264-270.

10. Prasad N, Gupta A, Sharma RK, Prasad KN, Gulati S, et al. (2003) Outcome of gram-positive and gram-negative peritonitis in patients on continuous ambulatory peritoneal dialysis: a single-center experience. Perit Dial Int 23: S144-147.

11. Bunke M, Brier ME, Golper TA (1994) Culture-negative CAPD peritonitis: the Network 9 Study. Adv Perit Dial 10: 174-178.

12. Jha V (2008) Peritoneal dialysis in India: current status and challenges. Perit Dial Int 28 Suppl 3: S36-41.

13. Sharma RK, Kumar J, Gupta A, Gulati S (2003) Peritoneal infection in acute intermittent peritoneal dialysis. Ren Fail 25: 975-980.

14. Keithi-Reddy SR, Gupta KL, Jha V, Sud K, Singh SK, et al. (2007) Spectrum and sensitivity pattern of gram-negative organisms causing CAPD peritonitis in India. Perit Dial Int 27: 205-207.

15. Davies SJ, Bryan J, Phillips L, Russell G (1996) Longitudinal changes in peritoneal kinetics: the effects of peritoneal dialysis and peritonitis. Nephrol Dial Transplant 11: 498-506.

16. Troidle L, Gorban-Brennan N, Kliger A, Finkelstein F (1998) Differing outcomes of gram-positive and gram-negative peritonitis. Am J Kidney Dis 32: 623-628. 
Citation: Abraham G, Gupta A, Prasad KN, Rohit A, Billa V, et al. (2016) Microbiology, Clinical Spectrum and Outcome of Peritonitis in Patients Undergoing Peritoneal Dialysis in India: Results from a Multicentric, Observational Study. J Trop Dis 4: 213. doi:10.4172/2329-891X. 1000213

Page 8 of 8

17. Lo WK, Chan CY, Cheng SW, Poon JF, Chan DT, et al. (1996) A prospective randomized control study of oral nystatin prophylaxis for Candida peritonitis complicating continuous ambulatory peritoneal dialysis. Am J Kidney Dis 28: 549-552.

18. Ghali JR, Bannister KM, Brown FG, Rosman JB, Wiggins KJ, et al. (2011) Microbiology and outcomes of peritonitis in Australian peritoneal dialysis patients. Perit Dial Int 31: 651-662.

19. Barretti P, Doles JV, Pinotti DG, El Dib RP (2015) Evidence-based medicine: An update on treatments for peritoneal dialysis-related peritonitis. World J Nephrol 4: 287-294.

20. Johnson CA, Zimmerman SW, Rogge M (1984) The pharmacokinetics of antibiotics used to treat peritoneal dialysis-associated peritonitis. Am J Kidney Dis 4: 3-17.

21. Santoianni JE, Predari SC, VerónD, Zucchini A, De Paulis AN (2008) A 15 year - review of peritoneal dialysis related peritonitis: microbiological trends and patterns of infections in a teaching hospital in Argentina. Rev Argent Microbiol. 40: 17-23.

22. Khairullah Q, Provenzano R, Tayeb J, Ahmad A, Balakrishnan R, et al. (2002) Comparison of vancomycin versus cefazolin as initial therapy for peritonitis in peritoneal dialysis patients. Perit Dial Int 22: 339-344.

23. Jörres A (2012) Novel peritoneal dialysis solutions--what are the clinical implications? Blood Purif 33: 153-159.

24. Kim DK, Yoo TH, Ryu DR, Xu ZJ, Kim HJ, et al. (2004). Changes in causative organisms and their antimicrobial susceptibilities in CAPD peritonitis: a single center's experience over one decade. Perit Dial Int 24: 424-32.

25. Marrie TJ, Noble MA, Costerton JW (1983) Examination of the morphology of bacteria adhering to peritoneal dialysis catheters by scanning and transmission electron microscopy. J Clin Microbiol. 18: 1388-1398. 